\title{
Silencing of ITGB6 inhibits the progression of cervical carcinoma via regulating JAK/STAT3 signaling pathway
}

\author{
Xiaoxia Zheng, Yanan Zhu, Xiaoping Wang, Yanmei Hou, Yingji Fang \\ Jinan Maternity and Child Care Hospital Affiliated to Shandong First Medical University/Jinan Maternity and Child Care Hospital Affiliated, Jinan, \\ China \\ Contributions: (I) Conception and design: Y Fang; (II) Administrative support: Y Hou; (III) Provision of study materials or patients: X Zheng, Y Zhu, \\ X Wang; (IV) Collection and assembly of data: X Zheng, Y Zhu, X Wang; (V) Data analysis and interpretation: X Zheng, Y Zhu, X Wang; (VI) \\ Manuscript writing: All authors; (VII) Final approval of manuscript: All authors. \\ Correspondence to: Yingji Fang. Jinan Maternity and Child Care Hospital Affiliated to Shandong First Medical University/Jinan Maternity and Child \\ Care Hospital Affiliated, No. 2 Jianguo Xiaojingsan Road, Jinan 250001, China. Email: yingji1972@126.com.
}

Background: Integrin $\beta 6$ (ITGB6), a key submonomer of integrin $\alpha v \beta 6$, plays an important role in epithelial-to-mesenchymal transition (EMT), wound healing, epithelial-derived tumor growth, fibrosis, and epithelial repair. However, the role of ITGB6 in cervical carcinoma (CC) remains elusive.

Methods: The expression levels of ITGB6 in CC tissues and cell lines were determined using quantitative real-time polymerase chain reaction (qRT-PCR). The cell viability, proliferation, apoptosis, migration, and invasion were evaluated by Cell Counting Kit-8 (CCK-8), colony-forming, flow cytometry, and Transwell assay, respectively. The expression of related proteins, including EMT markers and the Janus kinase/signal transducer and activator of transcription (JAK/STAT3) signaling markers, were detected using western blotting.

Results: The ITGB6 expression in CC tissues and cells (C-33A, Hela, SiHa, and Caski) was remarkably higher than that in paracarcinoma tissues and ECT1/E6E7 cells. The data from The Cancer Genome Atlas (TCGA) data set suggested that patients with CC with high ITGB6 expression showed poorer overall survival (OS). Compared with the empty transfection group (si-NC), si-ITGB6 restrained the proliferation, migration, and invasion of $\mathrm{SiHa}$ and Hela cells, while promoting cell apoptosis. si-ITGB6 suppression decreased the expression of Snail, vimentin, and N-cadherin, while increasing E-cadherin expression. Further research showed that si-ITGB6 reduced p-JAK1/JAK1, p-JAK2/JAK2, and p-STAT3/STAT3 expression in the JAK/STAT3 signaling pathway. Interestingly, proliferation, migration, invasion, and the expressions of the molecular markers of the JAK/STAT3 signaling pathway and EMT pathway induced by ITGB6 were altered by RO8191 (JAK/STAT3 pathway activator). Furthermore, the protein expression levels of Snail, vimentin, N-cadherin, p-STAT3/STAT3, p-JAK1/JAK1, and p-JAK2/JAK2 in tumor tissues were higher than those in adjacent normal tissue, while the expression level of E-cadherin was downregulated in tumor tissues.

Conclusions: Silencing of ITGB6 restrains cell proliferation, migration and invasion, and promotes apoptosis in CC by inhibiting JAK/STAT signaling pathways. Thus, ITGB6 may perhaps be a new and useful candidate target for treating CC.

Keywords: Cervical carcinoma (CC); ITGB6; proliferation; invasion; JAK/STAT signaling pathway

Submitted Mar 22, 2021. Accepted for publication Apr 29, 2021.

doi: 10.21037/atm-21-1669

View this article at: http://dx.doi.org/10.21037/atm-21-1669 


\section{Introduction}

Cervical carcinoma (CC) is the second most prevalent gynecologic cancer following breast cancer in females across the world, with high morbidity and mortality $(1,2)$. According to statistics, CC affects about 500,000 women worldwide and results in more than 300,000 deaths each year (3). It originates from cervical squamous or glandular epithelial cells, and includes three major histotypes: squamous cell carcinoma (approximately $80-85 \%$ ), adenosquamous carcinoma and adenocarcinoma (4). After the combined therapeutic approaches including radiotherapy, chemotherapy, and surgery, the majority of females with early-stage tumors can be cured, while one-third of CC patients diagnosed with advanced malignancy still have poor prognosis as a result of tumor metastasis and recurrence (5). Therefore, investigation of the underlying molecular mechanisms involved in CC progression is highly necessary and may generate new therapeutic strategies to improve the overall survival (OS) of CC patients.

Integrin $\beta 6$ (ITGB6), firstly isolated from the epithelial cells of guinea pigs in 1990 (6), is a key submonomer of integrin $\alpha v \beta 6$ (7). It is exclusively expressed on surfaces of epithelial cells, upregulated during epithelial-tomesenchymal transition (EMT), and is involved in wound healing, epithelial-derived tumor growth, fibrosis, and epithelial repair $(8,9)$. Integrin $\alpha \mathrm{V} \beta 6$ has been found to be overexpressed in some squamous cell carcinoma types (10-12), especially in cervical squamous cell carcinoma (13), and has often been associated with tumor metastasis and invasion. As integrin $\alpha \mathrm{v} \beta 6$ expression relies on ITGB6 expression in the whole heterodimer (14), it is necessary to clarify the mechanisms underlying the regulation of ITGB6 expression to facilitate the identification of potential therapeutic targets for CC.

The Janus kinase/signal transducer and activator of transcription (JAK/STAT) signaling pathway arbitrates the transcription pathways of a variety of cytokines, and is widely involved in regulating cell proliferation, apoptosis, survival, differentiation, immune response, and other biological processes of many cancers related to CC $(15,16)$. Herein, considering the vital roles of JAK/STAT3 signaling pathway, we speculated whether the silencing of ITGB6 could restrain proliferation, migration, and invasion and induce apoptosis of CC cells via modulating JAK/STAT3 signaling pathway.

We present the following article in accordance with the MDAR reporting checklist (available at http://dx.doi. org/10.21037/atm-21-1669).

\section{Methods}

\section{Patient specimens}

A total of 25 tumor tissues and paired adjacent normal tissues were collected from CC patients (female, age range of 25-60 years). All specimens were quickly frozen and stored in liquid nitrogen for further use. No patients underwent preoperative radiotherapy or chemotherapy before surgery. The study was approved by the Ethics and Research Committees of Jinan Maternity and Child Care Hospital Affiliated to Shandong First Medical University/ Jinan Maternity and Child Care Hospital Affiliated (approval number: 2019-1-026), and all patients completed written informed consent. All procedures performed in this study involving human participants were in accordance with the Declaration of Helsinki (as revised in 2013).

\section{Prediction of ITGB6 expression in CC}

The ITGB6 expression in CC tissue and normal tissue were retrieved by FireBrowse (http://firebrowse.org/) from The Cancer Genome Atlas (TCGA) biolinks R/Bioconductor software package. The transcripts per million (TPM) value for ITGB6 was used as the gene expression level. The expression levels of ITGB6 gene at different nodal stages of CC were also analyzed using TCGA data. The potential effect of ITGB6 on OS was analyzed by using the KaplanMeier method.

\section{Cell culture}

Cell lines were obtained from the American Type Culture Collection (ATCC; Manassas, VA, USA). The human CC cell lines, C-33A, Hela, SiHa, and Caski were maintained in Dulbecco's Modified Eagle's Medium (DMEM; Invitrogen, Carlsbad, CA, USA). The normal human cervical immortalized squamous cell line ECT1/E6E7 was cultured in RPMI 1640 medium (Gibco, Grand Island, NY, USA). All media were mixed with $10 \%$ of fetal bovine serum (FBS, Gibco), $50 \mu \mathrm{g} / \mathrm{mL}$ of streptomycin, $2 \mathrm{mM}$ of L-glutamine, and 50 units $/ \mathrm{ml}$ of penicillin $\mathrm{G}$ in a humid environment $\left(37^{\circ} \mathrm{C}\right)$ containing $5 \% \mathrm{CO}_{2}$.

\section{Cell transfection}

ITGB6 small interfering RNAs (siRNAs, Ribobio, 
Guangzhou, China) were used to specifically silence ITGB 6 expression in SiHa and Hela cells. The cells were transfected with siRNAs (si-NC; si1ITGB6, 5'-GAAAGAUUGUGUUAGUUAAGU-3', 5 ' - UUAACUAACACAAUCUUUCUA-3 '; si 2 ITGB6, 5'-GGAGAAUGUUGCUAAGCUAUU-3', 5'-UAGCUUAGCAACAUUCUCCUA-3') with the transfection reagent Lipofectamine 2000 (Invitrogen). After the confluence rate of SiHa and Hela cells in medium reached $70 \%, 5 \mu \mathrm{L}$ of siRNA and Lipofectamine 2000 reagents were gently mixed, and culture was continued for 4 hours in a humid environment containing $5 \% \mathrm{CO}_{2}$. The second day after Hela and SiHa cells were transfected, part of the si-NC and si1-ITGB6 cells were incubated in DMEM medium with $20 \mu \mathrm{M}$ of RO8191 and delineated as the si-NC + RO8191 group and si1-ITGB6 + RO8191 group, respectively. The cells were then used for the further experiments after at least 48 hours of incubation.

\section{Quantitative real-time polymerase chain reaction}

Total RNA was extracted from cells and tissues by TRIzol reagent (Invitrogen). The reverse transcription of total RNA to cDNA was performed with PrimeScript RT reagent Kit (TaKaRa, Japan). SYBR Green Real-Time PCR Master Mix (Thermo Fisher Scientific, USA) was applied to perform quantitative real-time polymerase chain reaction (qRTPCR) with an Applied Biosystems 7500 Sequence Detection system (ABI, USA). GAPDH was an internal control and chosen for normalizing, and the $2^{-\Delta \Delta \mathrm{Ct}}$ method was used to calculate the relative expression of genes. The sequences of the primers used in this study were listed as follows: ITGB6, 5'-ACTGGCCAGCTACTTACTGTG-3' (forward) and 5'-TTTTGGGGTTGTGACTTCACTG-3' (reverse); GAPDH, 5'-CACCAGGTGGTCTCCTCTGA-3' (forward) and 5'-TTGTCAGGGCCCTTTTTCTGA-3' (reverse).

\section{Immunobistochemical analysis}

The CC tissues and paired adjacent normal tissues from 25 patients, fixed by formalin and embedded paraffin, were cut into $4-\mu \mathrm{m}$ thick slices. The tissues were dehydrated, and xylene was removed by an ascending series of ethanol. Subsequently, the slides were incubated with a rabbit polyclonal anti-ITGB6 antibody (ab233519, 1:100, Abcam, USA) overnight at $4{ }^{\circ} \mathrm{C}$. After this, the sections were incubated with secondary antibodies at room temperature for 1 hour. After each step above, the tissues were washed with phosphate-buffered saline (PBS) for 5 minutes 3 times. Finally, sections were developed with 3,3'-diaminobenzidine (DAB, Sigma-Aldrich, St. Louis, MO, USA) for 30 minutes, counterstained with hematoxylin, and observed under microscope.

\section{Cell Counting Kit-8 assay}

The viability of cells cultured for $0,24,48$, and 72 hours was examined by Cell Counting Kit-8 (CCK-8; Dojindo, Japan). The absorbance at $450 \mathrm{~nm}$ was measured by a Thermomax microplate reader (Molecular Devices, CA, USA).

\section{Colony formation assay}

The cell proliferation was detected by colony-formation assay. After transfection for 48 hours, cells were seeded in a 6-well plate with complete medium for 2 weeks, which was replaced every 3 days. The colonies with more than 50 cells were then fixed with $4 \%$ formaldehyde for 15 minutes at $37^{\circ} \mathrm{C}$, subsequently stained with crystal violet for 10-20 minutes, and counted under the microscope. Finally, the data obtained from five stochastic fields were used for statistical analysis.

\section{Cell apoptosis assay}

An AnnexinV-PI kit (Beyotime, Nantong, China) was used for staining, and flow cytometry was used to detect cell apoptosis. Briefly, the harvested cells were flushed with PBS and promptly resuspended in $500 \mu \mathrm{L}$ of conjugate buffer. Then, $10 \mu \mathrm{L}$ of Annexin $\mathrm{V}$ - FITC and $10 \mu \mathrm{L}$ of propidium iodide (PI) were added to the buffer. A FACScan flow cytometer (BD Biosciences, CA, USA) was used to analyze cell apoptosis.

\section{Transwell assay}

For the Transwell assay, 24-well Transwell chambers with and without Matrigel (BD Pharmingen, CA, USA) were respectively used to detect the migration ability and invasion ability of SiHa and Hela cells. Briefly, $5 \times 10^{4}$ transfected cells $(100 \mu \mathrm{L})$ cultured in serum-free DMEM or DMEM containing $10 \%$ FBS were added to the upper transwell chamber $(8-\mu \mathrm{m}$ pore size) and lower chamber. After 24 hours, cells migrating or invading to the lower 
surface were immobilized with $5 \%$ glutaraldehyde, which was followed by staining with $0.2 \%$ crystal violet (Qianchen Biotechnology, Shanghai, China). Finally, the cells were counted in five random fields using an optical microscope (Nikon, Tokyo, Japan).

\section{Western blotting}

The total proteins from cells and tissues were extracted using radio immunoprecipitation assay (RIPA) buffer (Sigma-Aldrich). The density of total proteins was detected using a BCA Protein Assay kit (Takara). Next, proteins were separated with $10 \%$ sodium lauryl sulfate-polyacrylamide gels (SDS-PAGE) and quickly moved to polyvinylidene difluoride (PVDF) membranes (Thermo Fisher Scientific). The membrane was clogged with $5 \%$ nonfat dry milk, and mixed with primary antibodies against Snail [3879; 1:1,000; Cell Signaling Technology (CST), Danvers, MA, USA], vimentin (5741, 1:1,000, CST), N-cadherin (sc-8424; 1:1,000; Santa Cruz Biotechnology, CA, USA), E-cadherin (sc-71008, 1:1,000, Santa Cruz Biotechnology), JAK1 (29261, 1:1,000, CST), p-JAK1 (74129, 1:1,000, CST), JAK2 (3230, 1:1,000, CST), p-JAK2 (4406, 1:1,000, CST), p-STAT3 (52075, 1:1,000, CST), STAT3 (30835, 1:1,000, CST), and GAPDH $(5174,1: 1,000$, CST) overnight at $4{ }^{\circ} \mathrm{C}$. On day 2 , the proteins were incubated with horse radish peroxidase (HRP)-labeled secondary antibody (7074, 1:1,000, CST) for 45 minutes at room temperature. The intensity of protein expression was measured by an enhanced chemiluminescence reagent (Thermo Fisher Scientific) with GAPDH being used as the control.

\section{Gene set enrichment analysis}

To gain insight into the biological processes and predict the potential signal pathways of ITGB6 expression in CC, we performed an ITGB6 analysis using gene set enrichment analysis (GSEA) software that was obtained from the Broad Institute website (http://software.broadinstitute.org/gsea/ index.jsp). Gene expression data of CC were obtained from TCGA database.

\section{Statistical analysis}

All data were analyzed by GraphPad Prism 7. All the above experiments were conducted in triplicate technical replicates, and all data are expressed as mean \pm standard deviation (SD). Differences between two groups were determined by Student's $t$ test. Differences among multiple groups were determined by one-way analysis of variance (ANOVA). A P value less than 0.05 was considered to be statistically significant.

\section{Results}

\section{ITGB6 was overexpressed and predicted poor survival in CC patients}

TCGA cohort data sets showed that ITGB6 expression in CC tumor tissues and metastasis CC were both significantly higher compared with that in normal cervical tissues (Figure 1A,B). Additionally, CC patients with high ITGB6 expression showed poorer OS than patients with low ITGB6 expression (Figure 1C). We further examined ITGB6 expression in tumor tissues and normal tissues using qRT-PCR and immunohistochemistry (IHC), respectively. The results indicated that ITGB6 expression was upregulated in tumor tissues $(\mathrm{P}<0.01$, Figure $1 D, E)$.

\section{Silencing of ITGB6 suppressed cell proliferation and promoted apoptosis in CC cells}

The messenger RNA (mRNA) expression of ITGB6 in CC cells and normal cells was measured by qRT-PCR. In comparison with ECT1/E6E7 cells, tumors cell lines showed a higher expression level of ITGB6, especially the $\mathrm{SiHa}$ and Hela cells $(\mathrm{P}<0.01$, Figure $2 A)$. Thus, specific siRNAs against ITGB6 (si1-ITGB6 and si2-ITGB6) were first applied to restrain ITGB6 expression in $\mathrm{SiHa}$ and Hela cells. As the data presented in Figure $2 B$ indicate, the CC cell lines ( $\mathrm{SiHa}$ and Hela) transfected with ITGB6 siRNAs showed lower ITGB6 expression levels than did the cells transfected with negative control siRNAs (si-NC). To more intensively evaluate the roles of ITGB6 on CC development, the cell viability of $\mathrm{SiHa}$ and HeLa cells was detected using CKK-8 assay. The results demonstrated that si-ITGB6 markedly impaired the viability of both $\mathrm{SiHa}$ and Hela cells $(\mathrm{P}<0.01$, Figure $2 C)$. Analogously, the cell colonyforming assays clearly showed that si-ITGB6 significantly reduced cell colony number in both $\mathrm{SiHa}$ and Hela cells $(\mathrm{P}<0.01$, Figure 2D). Furthermore, through comparison with the si-NC group, increased apoptosis was observed after silencing ITGB6 in SiHa and Hela cells (Figure 2E). 
A

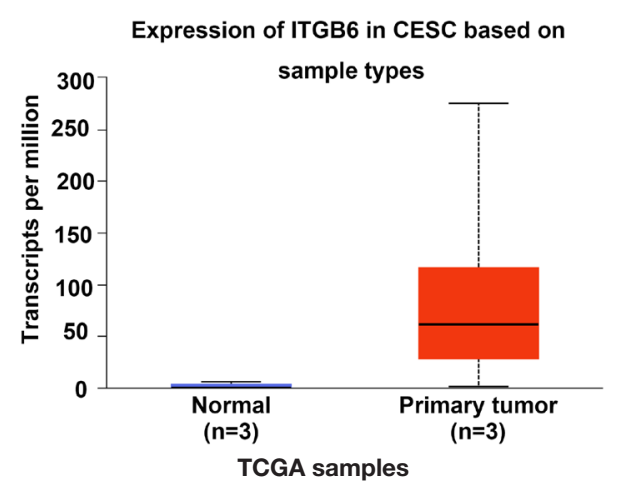

D

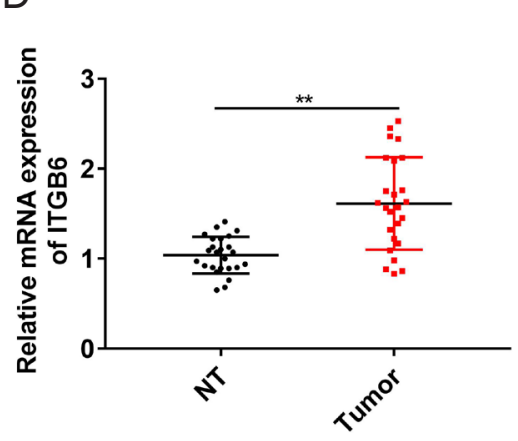

B

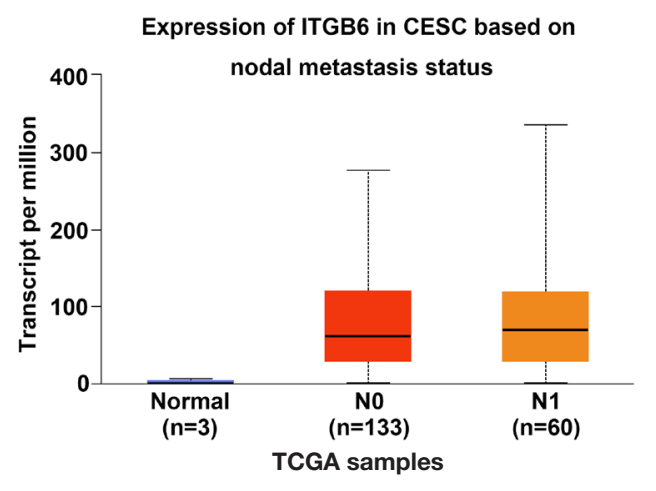

E
C
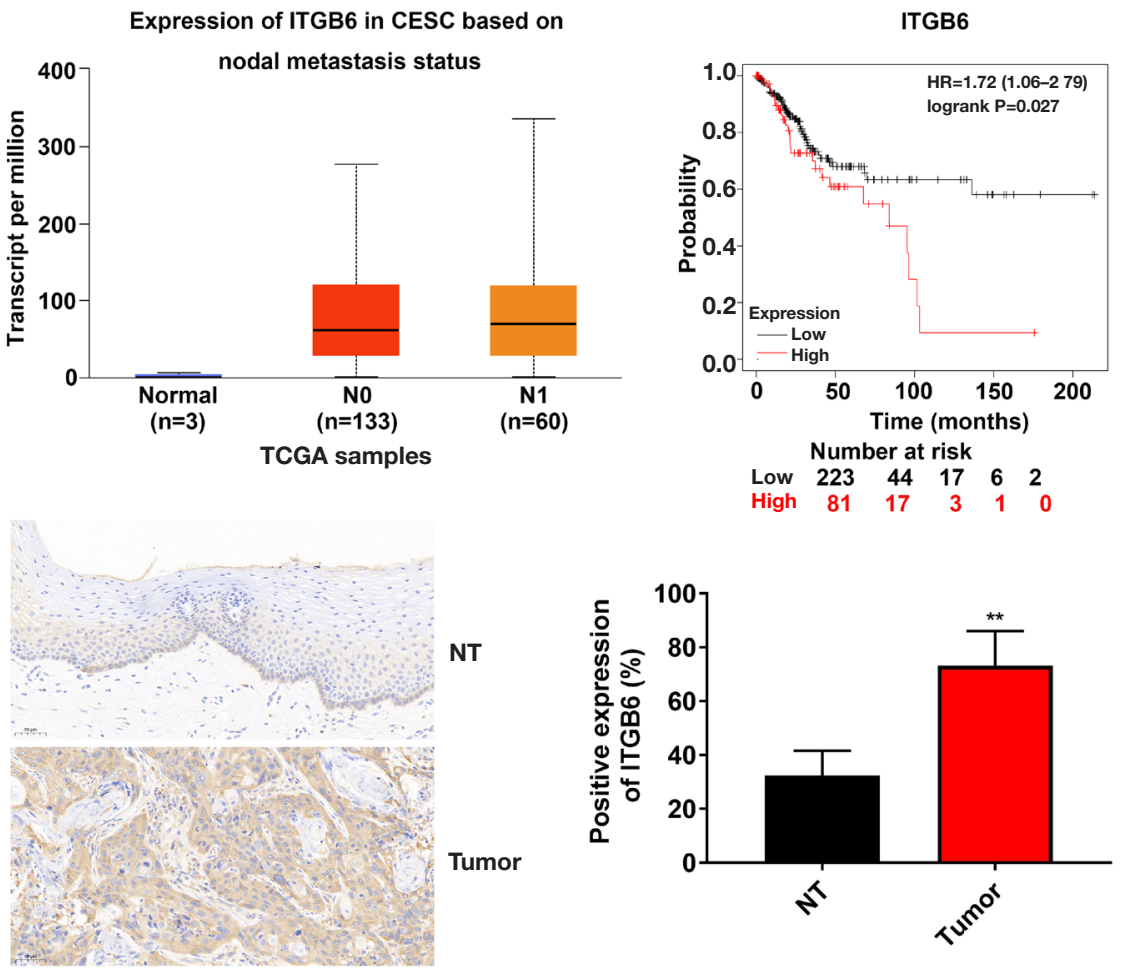

Figure 1 ITGB6 was upregulated in CC. (A,B) TCGA data sets showed ITGB6 expression was higher in tumor tissues than in normal tissues; (C) Kaplan-Meier survival plots demonstrated that higher ITGB6 abundance correlated with a poorer overall survival according to TCGA database; (D,E) The expression of ITGB6 in 25 CC tumor tissues and normal tissues was examined using qRT-PCR and IHC (magnification: $100 \times$ ), respectively. ${ }^{* *} \mathrm{P}<0.01$, compared with NT. ITGB6, Integrin $\beta 6$; CC, cervical carcinoma; TCGA, The Cancer Genome Atlas; N0, node negative; N1, node positive (1-3 positive nodes); NT, normal tissues; qRT-PCR, quantitative real-time polymerase chain reaction; IHC, immunohistochemistry.

\section{Silencing of ITGB6 suppressed migration and invasion of CC cells}

To investigate the potential effects of ITGB6 on the migration and invasion capabilities of CC cells, Transwell assays with $\mathrm{SiHa}$ and Hela cells were completed. As shown in Figure $3 A$ and $B$, si-ITGB6 markedly impaired the migration and invasion capability of $\mathrm{SiHa}$ and Hela cells in comparison with the si-NC group $(\mathrm{P}<0.01)$. Furthermore, the protein levels involved in EMT were evaluated by western blotting. According to results, both $\mathrm{SiHa}$ and Hela cells transfected with ITGB6 siRNAs exhibited a significant reduction in the expression of Snail, vimentin, and $\mathrm{N}$-cadherin, and a significant increase in the protein levels of E-cadherin $(\mathrm{P}<0.01$, Figure $3 C)$. Taken together, these results indicated that ITGB6 figured prominently in mediating the invasiveness and migration capability of CC cells, and that si-ITGB6 could exert its inhibitory effects of CC metastasis by affecting the EMT pathway.

\section{Silencing of ITGB6 attenuated FAK/STAT3 signaling patbway}

In order to explore the potential mechanisms of ITGB6 in CC progression, the GSEA analysis was used to predict the KEGG downstream pathway of ITGB6. The results showed that ITGB6 expression could activate the Hallmark IL6/JAK/STAT3 signaling (Figure 4A). Then, we evaluated the alteration of the proteins involved in the JAK/STAT3 signaling pathway by Western blotting assay. As shown in Figure 4B, the expression levels of p-STAT3/STAT3, p-JAK1/JAK1, and p-JAK2/JAK2 were all significant reduced after silencing of ITGB6 in SiHa and Hela cells $(\mathrm{P}<0.01)$. 


\section{A}

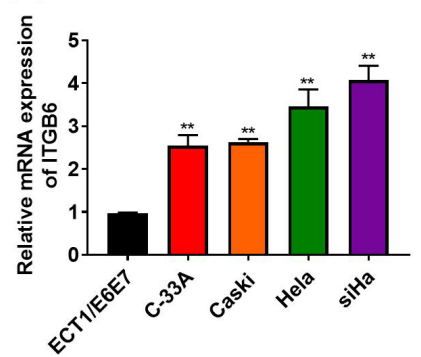

C

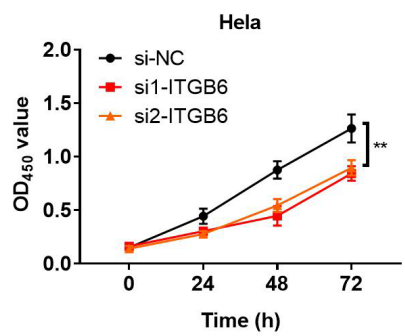

siHa

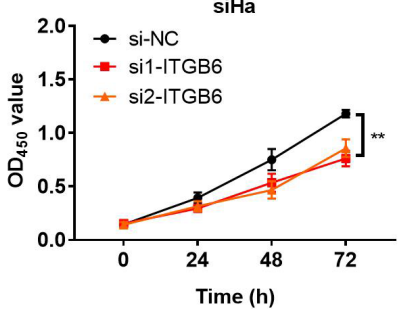

$\mathrm{B}$

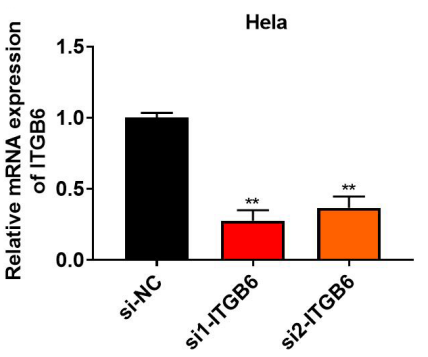

D
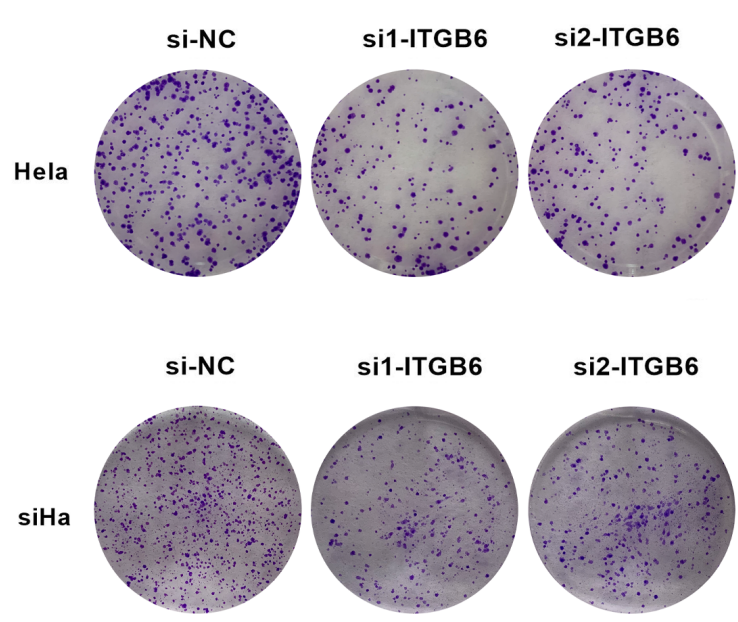

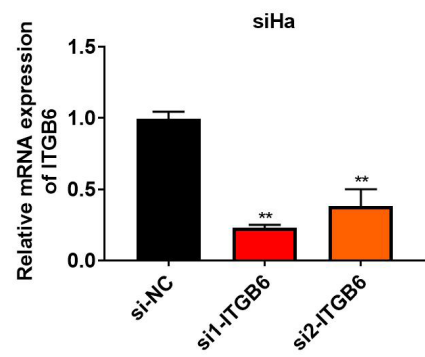

E
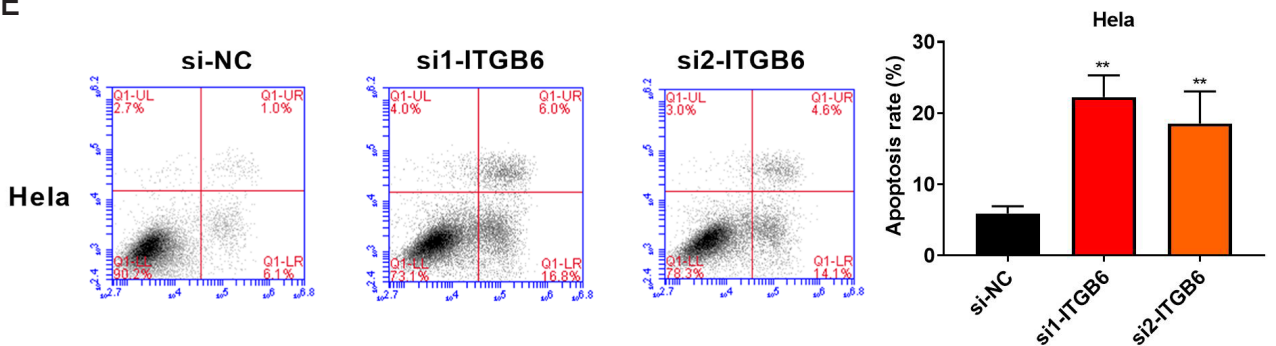

si-NC si1-ITGB6
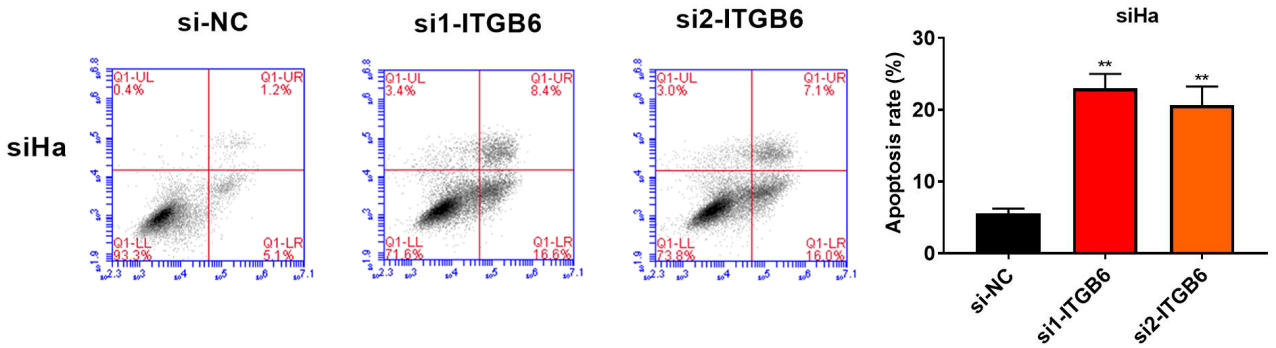

Figure 2 Silencing of ITGB6 suppressed cell proliferation and promoted apoptosis in CC cells. (A) The ITGB6 expression in CC cells lines (SiHa, Caski C-33A, and Hela) and human cervical immortalized squamous cell line (ECT1/E6E7) was tested by qRT-PCR; (B) relative mRNA expression levels of ITGB6 in SiHa and Hela cells transfected with ITGB6 siRNAs (si1-ITGB6 and si2-ITGB6) or NC siRNAs (si$\mathrm{NC})$; (C,D,E) the cell viability, proliferative capability, and cell apoptosis of transfected SiHa and Hela cells was evaluated by CKK-8, colonformation (crystal violet stain, magnification: $40 \times$ ), and flow cytometry assay, respectively. ${ }^{* *} \mathrm{P}<0.01$, compared with ECT1/E6E7, or the si-NC group. ITGB6, Integrin $\beta 6$; CC, cervical carcinoma; qRT-PCR, quantitative real-time polymerase chain reaction; si-NC, negative control siRNAs; CKK-8, Cell Counting Kit-8; OD, optical density. 
A

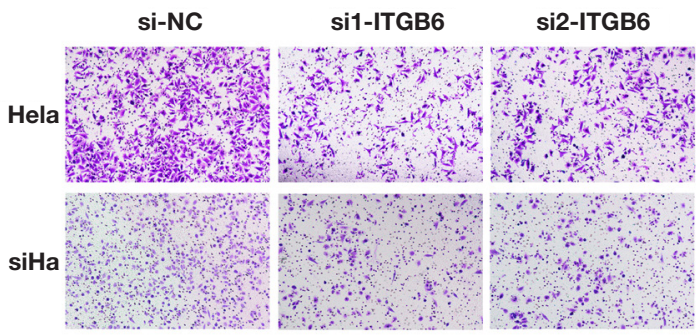

B

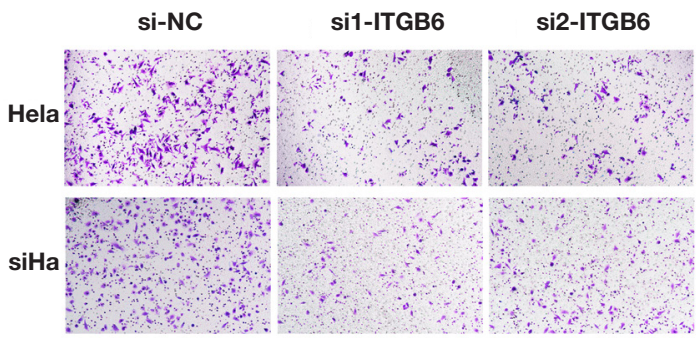

C

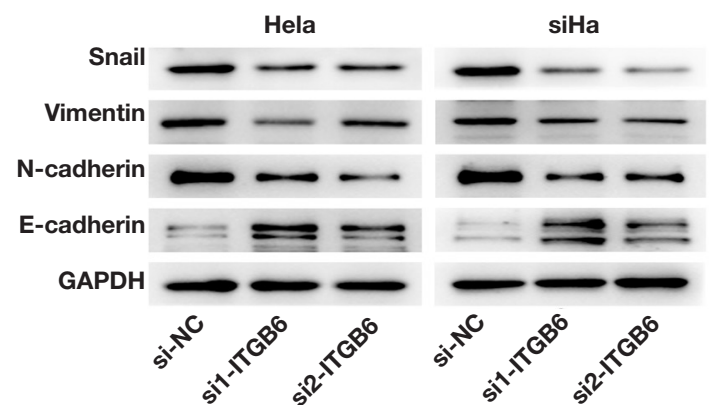

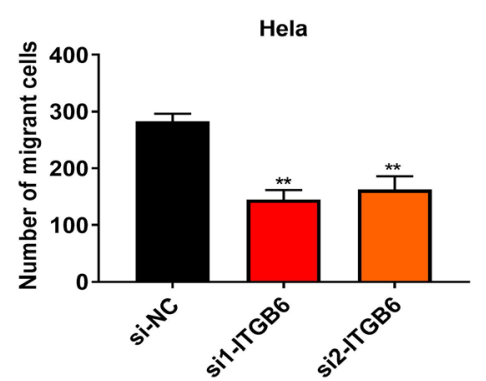
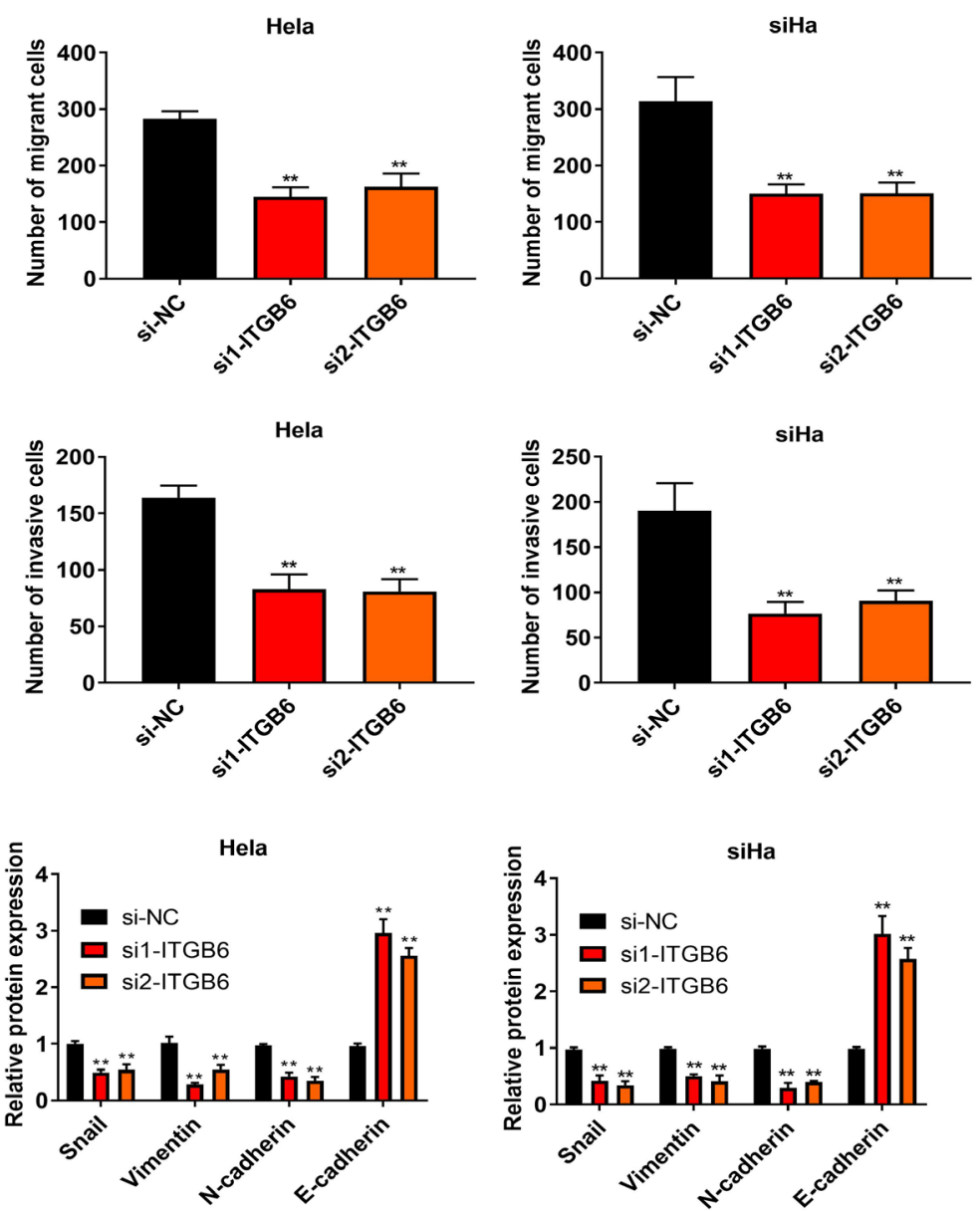

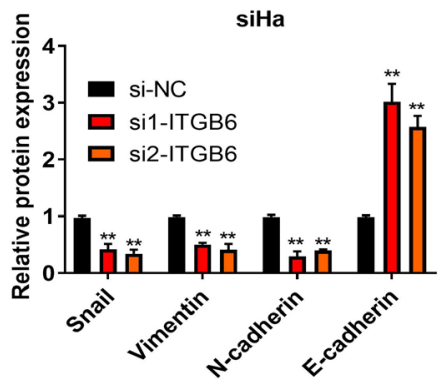

Figure 3 Silencing of ITGB6 inhibited the invasion, migration, and EMT of CC cells. (A,B) SiHa and Hela cell migration and invasion was tested by Transwell assays (crystal violet stain, magnification: 40x). (C) Western blotting assay was used to evaluate the protein expression levels of Snail, vimentin, $\mathrm{N}$-cadherin, and $\mathrm{E}$-cadherin in SiHa and Hela. ${ }^{* *} \mathrm{P}<0.01$, compared with the si-NC group. ITGB6, Integrin $\beta 6$; CC, cervical carcinoma; EMT, epithelial-to-mesenchymal transition; si-NC, negative control siRNAs.

\section{Silencing of ITGB6 repressed CC progression via regulating $\mathcal{F A K / S T A T 3}$ signaling pathway}

To further investigate the relationship between ITGB6 and JAK/STAT3 signaling pathway in CC progression, RO8191, an agonist of JAK/STAT3 signaling pathway, was used to treat Hela and $\mathrm{SiHa}$ cells. As exhibited in Figure 5A, the addition of RO8191 promoted the expression of p-STAT3/STAT3, p-JAK1/JAK1, and p-JAK2/JAK2 $(\mathrm{P}<0.01)$. si-ITGB6 markedly reduced the formation of cell clones, while the addition of RO8191 partially counteracted the inhibitory effect of si-ITGB6 on proliferation activity of Hela and SiHa cells $(\mathrm{P}<0.01$; Figure $5 B)$. Meanwhile, Transwell assay indicated that RO8191 could reverse the effect of si-ITGB6 on the migration and invasion of Hela and $\mathrm{SiHa}$ cells $(\mathrm{P}<0.01$; Figure $5 C, D)$. Furthermore, the protein expression of Snail, vimentin, and $\mathrm{N}$-cadherin downregulation and E-cadherin upregulation by si-ITGB6 was restored by RO8191 ( $\mathrm{P}<0.01$; Figure 5E). These data suggested that silencing of ITGB6 dampened the proliferation, invasion, and migration of CC cells via the inactivation of the JAK/STAT3 pathway. 


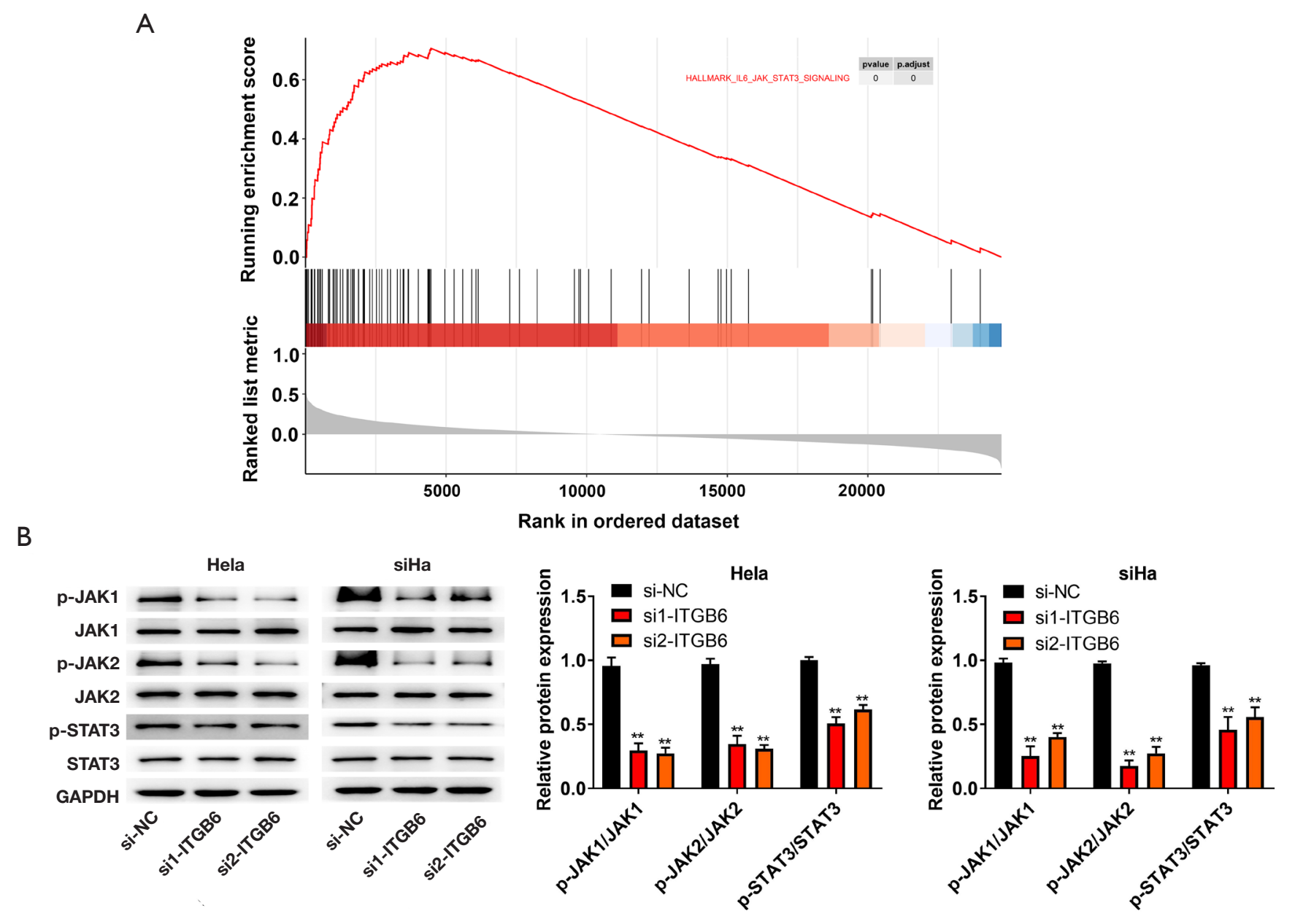

Figure 4 Silencing of ITGB6 inhibited the JAK/STAT3 signaling pathway in CC cells. (A) GSEA analysis found that ITGB6 expression was positively correlated with HALLMARK IL6/JAK/STAT3 signaling pathway; (B) Western blotting assay was applied to detect the JAK/STAT3 signaling pathway-related proteins (JAK1, p-JAK1, JAK2, p-JAK2, p-STAT3, and STAT3) in SiHa and Hela cells. ** $\mathrm{P}<0.01$, compared with the si-NC group. ITGB6, Integrin $\beta 6$; CC, cervical carcinoma; GSEA, gene set enrichment analysis; IL6/JAK/STAT3, Interleukin-6/janus kinase/signal transducer and activator of transcription; si-NC, negative control siRNAs.

\section{The expression level of EMT-related proteins and IL6/ JAK/STAT3 patbway-related proteins in tumor tissues and paired adjacent normal tissues}

Furthermore, we detected the expression level of EMTrelated proteins and IL6/JAK/STAT3 pathway-related proteins in tumor tissues and paired adjacent normal tissues using Western blotting. The results showed that the protein expression levels of Snail, vimentin, and N-cadherin were higher than those in adjacent normal tissue, while the expression level of $\mathrm{E}$-cadherin was downregulated in tumor tissues $(\mathrm{P}<0.01$; Figure $6 A)$. The expression levels of p-STAT3/STAT3, p-JAK1/JAK1, and p-JAK2/JAK2 in tumor tissue were also significantly higher than those in adjacent normal tissue $(\mathrm{P}<0.01$, Figure $6 B)$.

\section{Discussion}

$\mathrm{CC}$ is a malignant tumor which poses a serious threat to the health of women. Its occurrence is a multistep process involving virus infection, immune factors, proto-oncogenes and tumor-suppressor genes. The long-term prognosis of CC remains poor, and the 5-year survival rate is than $10 \%$ (17). It is generally believed that human papillomavirus (HPV) infection is the main cause of CC (18). However, an increasing number of studies are reporting that HPV is not 


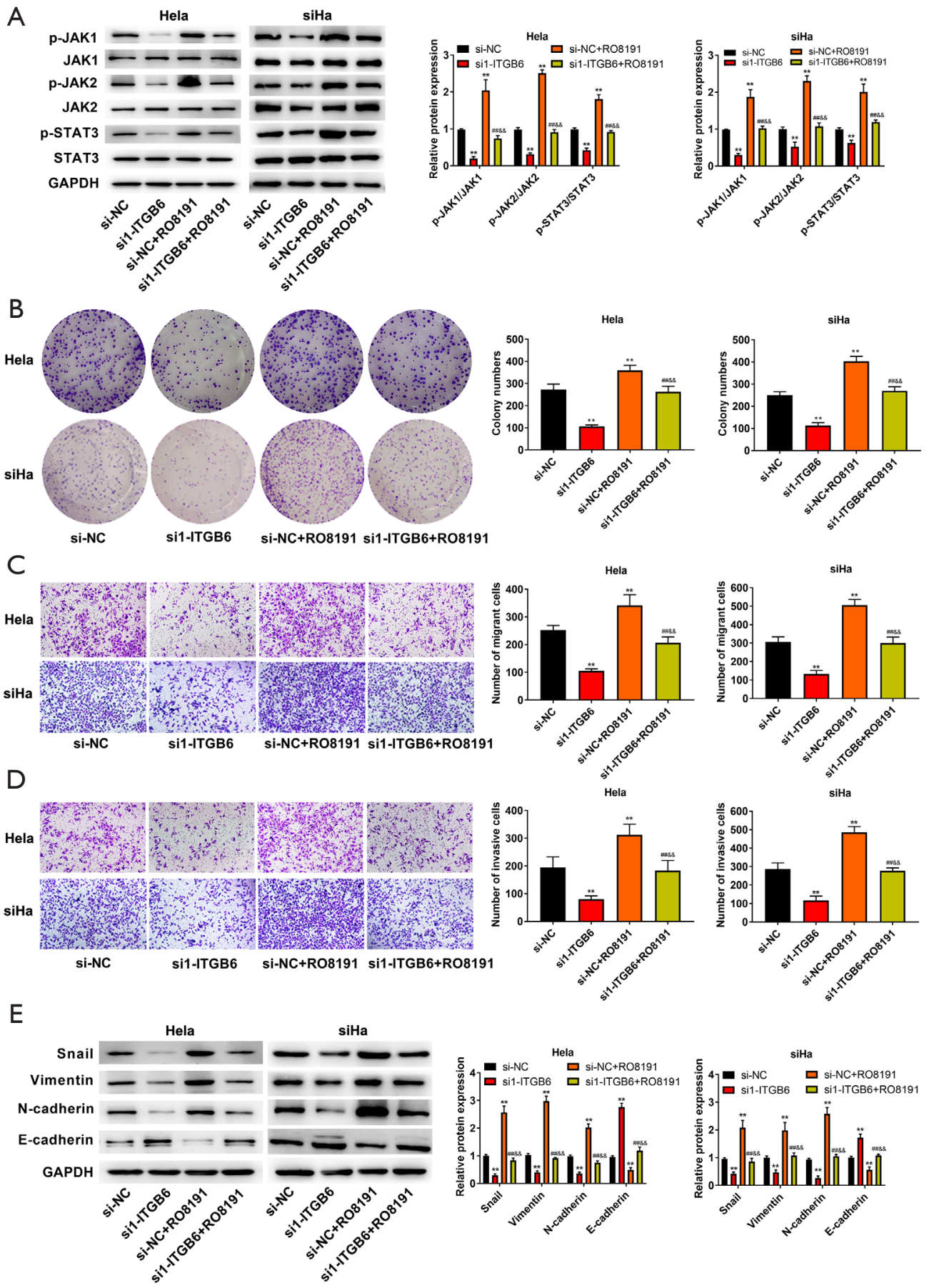

Figure 5 Silencing of ITGB6 suppressed CC progression via regulating JAK-STAT3 signaling. (A) Western blotting assay was applied to detect the JAK/STAT3 signaling pathway-related proteins (JAK1, p-JAK1, JAK2, p-JAK2, p-STAT3, and STAT3) in Hela and SiHa cells; (B) The cell proliferation of Hela and SiHa cells was evaluated by colony formation (crystal violet stain, magnification: 40x); (C,D) Transwell assay was used to evaluate the migration and invasion of Hela and SiHa cells (crystal violet stain, magnification: 40x); (E) Western blotting assay was applied to detect the EMT-related proteins (Snail, vimentin, N-cadherin, and E-cadherin) in Hela and SiHa cells. ${ }^{* *} \mathrm{P}<0.01$, compared with si-NC; ${ }^{\# \#} \mathrm{P}<0.01$, compared with the si1-ITGB6 group, ${ }^{\text {\&\& }} \mathrm{P}<0.01$, compared with the si-NC + RO8191 group. ITGB6, Integrin $\beta 6$; CC, cervical carcinoma; JAK/STAT3, Janus kinase/signal transducer and activator of transcription; EMT, epithelial-tomesenchymal transition; si-NC, negative control siRNAs. 
A

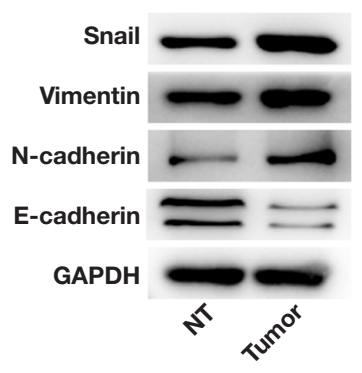

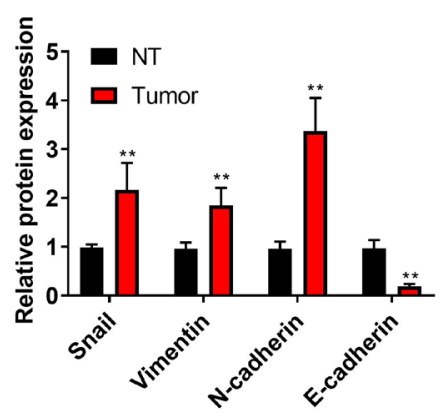

B

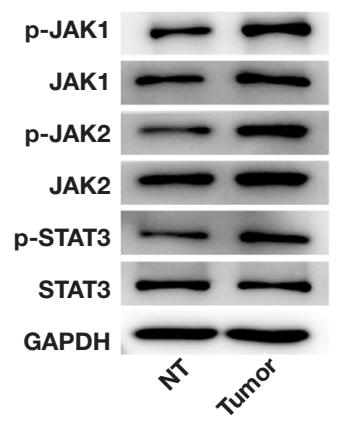

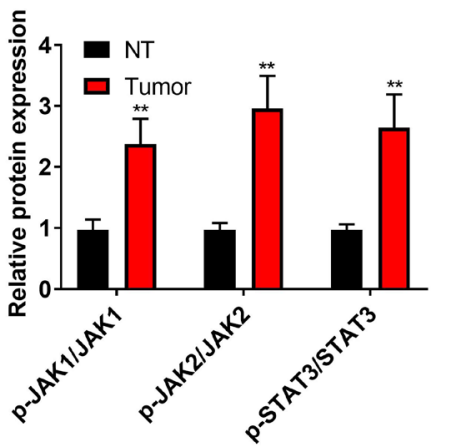

Figure 6 The expression level of EMT-related proteins and JAK/STAT3 pathway-related proteins in tumor tissues and paired adjacent normal tissues. (A) The expression levels of EMT-related proteins (Snail, vimentin, E-cadherin, and N-cadherin) in 25 CC tumor tissues and normal tissues were examined using Western blotting. (B) The expression levels of JAK/STAT3 pathway-related proteins (p-STAT3, STAT3, p-JAK1, JAK1, p-JAK2, and JAK2) in 25 CC tumor tissues and normal tissues were detected using Western blotting. ${ }^{* *} \mathrm{P}<0.01$, compared with NT. CC, cervical carcinoma; NT, normal tissues; EMT, epithelial-to-mesenchymal transition; JAK/STAT3, Janus kinase/ signal transducer and activator of transcription.

the only cause of CC development, while the limited HPVdirected therapy on CC has not been very effective (19). Recently, genetic alterations have been proven to be closely related to cancer progression, and these may serve as new targets or biomarkers in the treatment of CC.

The ITGB6 gene, located on human chromosomes of $2 \mathrm{q} 24-\mathrm{q} 31$ (20), determines the cellular expression of integrin $\alpha v \beta 6$. It is a liposome drug delivery target and has specific tumor-interrelated properties, making a latent targeting receptor for cancer therapy (7). Recently, many studies have confirmed that integrin $\alpha v \beta 6$ or ITGB6 plays an important role in various cancers, including epithelial ovarian cancer, colorectal carcinoma and breast cancer (10,21-23). Furthermore, Hazelbag et al. reported that av $\beta 6$ overexpression is an unfavorable prognostic factor in CC and can predict the onset of metastatic disease (13). However, the mechanism of ITGB6 in CC has not been extensively investigated. We thus aimed to characterize the expression pattern and clinical significances of ITGB6 in CC tissues by taking full advantage the of publicly available microarray data sets in TCGA. We found that ITGB6 expression was expressively upregulated in CC or metastatic CC and was associated with poor OS. Then, using the tissue samples from our hospital, we conducted qRT-PCR and IHC to further confirm that ITGB6 is highly expressed in CC.

Metastasis and invasion are the crucial biological features of malignant tumor and the pathological basis of tumor recurrence and deterioration. One study has reported that in colorectal carcinoma cells, ITGB6 can promote its own expression via autocrine signaling, induce chemotherapy resistance, cell proliferation, migration, and invasion, and inhibit apoptosis with its unique cytoplasmic tail; meanwhile, other integrins have no such effect (21). The anomalous induction of EMT is of vital importance in the metastasis and invasion of tumors. This study found that the protein expression levels of Snail, vimentin, and $\mathrm{N}$-cadherin in CC were higher than those in adjacent normal tissue, while the expression level of E-cadherin was downregulated in CC. The $\alpha v \beta 6$ integrin can promote the activation of autocrine tumor necrosis factor beta (TGF- $\beta$ ) that sustains EMT (24). One study has reported that EMT induces a significant increase in integrin $\alpha v \beta 6$ expression in colon cancer, which is reliant on ITGB6-mediated transcriptional activation (25). Sun et al. also have reported that ITGB6 promotes EMT and metastasis of colorectal cancer cells to the liver (26). Until now, the specific regulatory effect and mechanism of ITGB6 on CC have not been clarified. In this study, we found that silencing ITGB6 could suppress cell proliferation, migration, and invasion, and promote apoptosis of both $\mathrm{SiHa}$ and Hela cells. Furthermore, the alterations of EMT-related proteins were detected, and interestingly, si-ITGB6 induced an increase of E-cadherin and a reduction of $\mathrm{N}$-cadherin, Snail, and vimentin levels in CC. Additionally, silencing of ITGB6 markedly suppressed the EMT pathway. These findings, which are the first of their kind, indicate that ITGB6 is likely a vital contributor to CC progression. 
The JAK/STAT signaling pathway is a cellular signaling pathway activated by the epidermal growth factor receptor, JAK, which mainly includes JAK and STAT families (27). Activation or inactivation of the JAK/STAT signaling pathway is associated with the occurrence of a variety of cancers including $\mathrm{CC}$, and is pivotal in promoting carcinogenesis (28). Interleukin-6 (IL-6) has been reported to regulate the JAK/STAT. JAK and STAT3 are the major proteins of JAK-STAT3 pathway, and activate signaling pathway by phosphorylation. In this study, we found that the expression levels of p-STAT3/STAT3, p-JAK1/ JAK1, and p-JAK2/JAK2 in CC were significantly higher than those in adjacent normal tissue. The GSEA analysis indicated that the high expression of ITGB6 is related to IL-6 and JAK/STAT3 signaling pathway. The levels of p-JAK1/JAK1, p-JAK2/JAK2, and p-STAT3/STAT3 were markedly reduced after ITGB6 was silenced. To further investigate the relationship between ITGB6 and JAK/ STAT3 signaling pathway in CC progression, RO8191 (agonist of JAK/STAT3 signaling pathway) was added. As expected, the addition of RO8191 reversed proliferation, migration, invasion, and the expression of EMT and JAK/ STAT3 pathway-related proteins induced by ITGB6.

\section{Conclusions}

This study is the first to investigate the function and regulation mechanism of ITGB6 in CC. Our research confirmed that the silencing of ITGB6 could restrain cell proliferation, migration, and invasion, and promote apoptosis in CC by inhibiting JAK/STAT signaling pathways. Thus, ITGB6 is likely to be a candidate target for CC treatment.

\section{Acknowledgments}

Funding: None.

\section{Footnote}

Reporting Checklist: The authors have completed the MDAR reporting checklist. Available at http://dx.doi.org/10.21037/ atm-21-1669

Data Sharing Statement: Available at http://dx.doi. org/10.21037/atm-21-1669

Conflicts of Interest: All authors have completed the ICMJE uniform disclosure form (available at http://dx.doi. org/10.21037/atm-21-1669). The authors have no conflicts of interest to declare.

Ethical Statement: The authors are accountable for all aspects of the work in ensuring that questions related to the accuracy or integrity of any part of the work are appropriately investigated and resolved. All procedures performed in this study involving human participants were in accordance with the Declaration of Helsinki (as revised in 2013). The study was approved by the Ethics and Research Committees of Jinan Maternity and Child Care Hospital Affiliated to Shandong First Medical University/ Jinan Maternity and Child Care Hospital Affiliated (approval number: 2019-1-026), and all patients completed written informed consent.

Open Access Statement: This is an Open Access article distributed in accordance with the Creative Commons Attribution-NonCommercial-NoDerivs 4.0 International License (CC BY-NC-ND 4.0), which permits the noncommercial replication and distribution of the article with the strict proviso that no changes or edits are made and the original work is properly cited (including links to both the formal publication through the relevant DOI and the license). See: https://creativecommons.org/licenses/by-nc-nd/4.0/.

\section{References}

1. Diefenbach D, Greten HJ, Efferth T. Genomic landscape analyses in cervical carcinoma and consequences for treatment. Curr Opin Pharmacol 2020;54:142-57.

2. Small W Jr, Bacon MA, Bajaj A, et al. Cervical cancer: A global health crisis. Cancer 2017;123:2404-12.

3. Cancer Genome Atlas Research Network; Albert Einstein College of Medicine; Analytical Biological Services, et al. Integrated genomic and molecular characterization of cervical cancer. Nature 2017;543:378-84.

4. Halle MK, Ojesina AI, Engerud H, et al. Clinicopathologic and molecular markers in cervical carcinoma: a prospective cohort study. Am J Obstet Gynecol 2017;217:432.e1-432.e17.

5. Kumar L, Gupta S. Integrating Chemotherapy in the Management of Cervical Cancer: A Critical Appraisal. Oncology 2016;91 Suppl 1:8-17.

6. Sheppard D, Rozzo C, Starr L, et al. Complete amino acid sequence of a novel integrin beta subunit (beta 6) identified in epithelial cells using the polymerase chain reaction. J 
Biol Chem 1990;265:11502-7.

7. Liang B, Shahbaz M, Wang Y, et al. Integrinbeta6-targeted immunoliposomes mediate tumor-specific drug delivery and enhance therapeutic efficacy in colon carcinoma. Clin Cancer Res 2015;21:1183-95.

8. Thomas GJ, Nystrom ML, Marshall JF. Alphavbeta6 integrin in wound healing and cancer of the oral cavity. J Oral Pathol Med 2006;35:1-10.

9. Breuss JM, Gallo J, DeLisser HM, et al. Expression of the beta 6 integrin subunit in development, neoplasia and tissue repair suggests a role in epithelial remodeling. J Cell Sci 1995;108:2241-51.

10. Ahmed N, Pansino F, Clyde R, et al. Overexpression of alpha(v)beta6 integrin in serous epithelial ovarian cancer regulates extracellular matrix degradation via the plasminogen activation cascade. Carcinogenesis 2002;23:237-44.

11. Hecht JL, Dolinski BM, Gardner HA, et al. Overexpression of the alphavbeta6 integrin in endometrial cancer. Appl Immunohistochem Mol Morphol 2008;16:543-7.

12. Ramos DM, But M, Regezi J, et al. Expression of integrin beta 6 enhances invasive behavior in oral squamous cell carcinoma. Matrix Biol 2002;21:297-307.

13. Hazelbag S, Kenter GG, Gorter A, et al. Overexpression of the alpha v beta 6 integrin in cervical squamous cell carcinoma is a prognostic factor for decreased survival. J Pathol 2007;212:316-24.

14. Bandyopadhyay A, Raghavan S. Defining the role of integrin alphavbeta6 in cancer. Curr Drug Targets 2009;10:645-52.

15. Jin W. Role of JAK/STAT3 Signaling in the Regulation of Metastasis, the Transition of Cancer Stem Cells, and Chemoresistance of Cancer by Epithelial-Mesenchymal Transition. Cells 2020;9:217.

16. Wang DW, You D, Dong J, et al. Knockdown of long non-coding RNA LINC00518 inhibits cervical cancer proliferation and metastasis by modulating JAK/STAT3 signaling. Eur Rev Med Pharmacol Sci 2019;23:496-506.

17. Xu Y, Zhu L, Zhu L, et al. Strain elastography as an early predictor of long-term prognosis in patients with locally advanced cervical cancers treated with concurrent chemoradiotherapy. Eur Radiol 2020;30:471-81.

18. Yuan Y, Cai X, Shen F, et al. HPV post-infection microenvironment and cervical cancer. Cancer Lett 2021;497:243-54.

19. Ouyang X, Hao X, Liu S, et al. Expression of Nup93 is associated with the proliferation, migration and invasion capacity of cervical cancer cells. Acta biochimica et biophysica Sinica 2019;51:1276-85.

20. Fernández-Ruiz E, Sanchez-Madrid F, Regional localization of the human integrin beta 6 gene (ITGB6) to chromosome 2q24-q31. Genomics 1994;21:638-40.

21. Bengs S, Becker E, Busenhart P, et al. beta6 -integrin serves as a novel serum tumor marker for colorectal carcinoma. Int J Cancer 2019;145:678-85.

22. Desai K, Nair MG, Prabhu JS, et al. High expression of integrin beta6 in association with the Rho-Rac pathway identifies a poor prognostic subgroup within HER2 amplified breast cancers. Cancer Med 2016;5:2000-11.

23. Moore KM, Thomas GJ, Duffy SW, et al. Therapeutic targeting of integrin $\alpha v \beta 6$ in breast cancer. J Natl Cancer Inst 2014;106:dju169.

24. Lee C, Lee C, Lee S, et al. The cytoplasmic extension of the integrin beta6 subunit regulates epithelial-tomesenchymal transition. Anticancer Res 2014;34:659-64.

25. Bates RC, Bellovin DI, Brown C, et al. Transcriptional activation of integrin beta6 during the epithelialmesenchymal transition defines a novel prognostic indicator of aggressive colon carcinoma. J Clin Invest 2005;115:339-47.

26. Sun Q, Shang Y, Sun F, et al. Interleukin-6 Promotes Epithelial-Mesenchymal Transition and Cell Invasion through Integrin beta6 Upregulation in Colorectal Cancer. Oxid Med Cell Longev 2020;2020:8032187.

27. O'Shea JJ, Schwartz DM, Villarino AV, et al. The JAKSTAT pathway: impact on human disease and therapeutic intervention. Annu Rev Med 2015;66:311-28.

28. Groner B, von Manstein V. Jak Stat signaling and cancer: Opportunities, benefits and side effects of targeted inhibition. Mol Cell Endocrinol 2017;451:1-14.

(English Language Editor: J. Gray)
Cite this article as: Zheng $\mathrm{X}$, Zhu Y, Wang X, Hou Y, Fang Y. Silencing of ITGB6 inhibits the progression of cervical carcinoma via regulating JAK/STAT3 signaling pathway. Ann Transl Med 2021;9(9):803. doi: 10.21037/atm-21-1669 